\title{
Muscle Activity of Lower Extremities for Normal Adults According to the Type of Chair and Posture During Sit-to-stand Movement
}

\author{
Sunghyoun $\mathrm{Cho}^{1}$ and Haewon Byeon ${ }^{2 *}$ \\ 1 Dept.t of Physical Therapy, Nambu University, Gwangju, South Korea, \\ geriatricpt1@naver.com \\ 2 Dept. of Speech Language Pathology \& Audiology, Nambu University, \\ Gwangju, South Korea, byeon@nambu.ac.kr
}

\begin{abstract}
The purpose of this study is to investigate the effect of sit to stand movement on the muscle activity of lower extremities muscles by the different type of chair and posture. Total 52 healthy adult between 19 and 26 were selected as subject for the study. The muscle activities of vastus lateralis, rectus femoris, vastus medialis, tibialis anterior and gastrocnemius by the different types of chair during sit to stand movement were measured by using surface electromyography WEMG. Sit to stand movement was performed by the different types of chair. For both fixed and wheeled chairs, rectus femoris and vastus lateralis showed a statistically significant difference ( $p<.05)$ when standing, while vastus medialis, rectus femoris, and vastus lateralis showed a statistically significant difference when sitting $(p<.05)$. Comparison of standing and sitting positions revealed that all muscle types except gastrocnemius showed a statistically significant difference. This study suggests that it can be useful for providing fundamental data regarding the muscle activation of lower limbs according to chair type.
\end{abstract}

Keywords: Sit to stand movement, Lower extremity muscle, Chair type, Posture

\section{Introduction}

The sit-to-stand (STS) movement is the most fundamental condition for other movements, as all movements, such as transferring from one position to another, walking, spinning, and climbing stairs, are possible only when someone is able to maintain a standing position [1]. Moreover, STS is a useful tool for evaluating muscle strength and the functional conditions of lower limbs without using special training or a device [2].

Sit to stand (STS) is a complex movement requiring appropriate posture control to change from a 3-point-based siting posture to 2-point-based standing posture [3]. The body center moves in the upward direction while maintaining balance from sitting to standing postures [4].

Position control during STS is related to dynamic balancing [5] and a balancing function is required to prevent falling that can be caused by a drastic change in the basal plane and acceleration of the center of the body [6]. During the STS movement, the center of gravity moves forward and the lower limbs extend, which causes the center of gravity to deviate from a stable condition. Schenkman et al. [7] classified STS movement into four stages. The first stage is the flexion-moment stage, wherein the weight is moved to form an early moment. The second stage is the moment-movement stage, wherein the person detaches the hip from the chair.

* Corresponding author 
The foot flexion of the ankle joint becomes maximal in this stage. The third stage is the extension stage, wherein the person stands up straight by completely extending the hip and knee joints. Finally, the fourth stage is the stabilizing stage, wherein the hip and knee joints are completely extended. This kind of classification has been widely used to describe motion analyses [8-10]. Standing from a sitting position requires a physical change in the horizontal and vertical directions. Here, important external conditions that can affect standing include foot location, early trunk location, motion speed, chair height, use of the arms, gender, age [11]. As such, a patient's STS ability can be evaluated for various objectives through a number of methods.

In evaluating the STS movement, the center of body speed and lower limb joint moments are important factors, which are used to measure how the angular motion of a joint in a given direction during all STS phases affects the entire body's linear movement [12]. A restriction of STS movement from a chair is related closely to degradation in balance [13] and it can also affect gait ability, as characterized by time delay of STS task performance, outer or inner side displacements of the center of mass, and asymmetrical weight loads [14].

Using the upper limbs during STS merely helps to maintain balance, rather than decrease the load on the lower limb joints [15]. Among the factors that interrupt STS movement, chair height has the most critical impact [16]

Studies on the STS movement can be largely divided into three categories: the motion analysis approach using a kinematic system, the muscle activation pattern analysis approach using an electromyogram signal analysis system, and the simultaneous approach of a kinetic analysis using a force plate. A study has used electromyography (EMG) to determine how muscle energy supports the human body against gravity, how muscle energy achieves goal movements, and how long healthy subjects and people with various diseases require rest [17]. As of now, studies on the evaluation of standing up from a chair are insufficient. Hence, this study attempts to examine how the STS movement according to chair type affects the muscle activity of the lower limbs.

\section{Methods}

\subsection{Subjects}

For analyzing the muscle activity of the five lower limb muscles (vastus lateralis, rectus femoris, vastus medialis, tibialis anterior, and gastrocnemius muscle), students in their 20s enrolled at University $\mathrm{N}$ located in Metropolitan City $\mathrm{G}$ in Korea were selected as research subjects. The subjects included 52 healthy adults in their 20s (26 men and 26 women) who have no anomalies in muscle strength and joint range of motion, so the experiment can be sufficiently applied.

Among 52 adults who participated in this study, 26 were men and 26 were women. The men's average age was $21.10 \pm 1.86$ years, average height was 174.96 $\pm 4.34 \mathrm{~cm}$, average weight was $69.23 \pm 10.11 \mathrm{~kg}$, and BMI was $22.61 \pm 3.15 \mathrm{~kg} / \mathrm{m}^{2}$. The women's average age was $20.54 \pm 1.27$ years, average height was $163.77 \pm 3.86$ $\mathrm{cm}$, average weight was $57.46 \pm 8.09 \mathrm{~kg}$, and BMI was $21.39 \pm 2.63 \mathrm{~kg} / \mathrm{m}^{2}(\mathrm{p}>.05)$. (Table 1). 
Table 1. General Characteristics of Subjects

$(\mathrm{M} \pm \mathrm{SD})$

\begin{tabular}{llll}
\hline Category & Male $(\mathbf{n}=\mathbf{2 6})$ & Female $(\mathbf{n}=\mathbf{2 6})$ & Total $(\mathbf{N}=\mathbf{5 2})$ \\
\hline Age $(\mathbf{y r})$ & $21.10 \pm 1.86$ & $20.54 \pm 1.27$ & $20.82 \pm 1.61$ \\
Height $(\mathbf{c m})$ & $174.96 \pm 4.34$ & $163.77 \pm 3.86$ & $169.37 \pm 6.96$ \\
Weight $(\mathbf{k g})$ & $69.23 \pm 10.11$ & $57.46 \pm 8.09$ & $63.35 \pm 10.84$ \\
BMI $\left(\mathbf{k g} / \mathbf{m}^{2}\right)$ & $22.61 \pm 3.15$ & $21.39 \pm 2.63$ & $22.00 \pm 2.94$ \\
\hline
\end{tabular}

$\mathrm{M} \pm \mathrm{SD}:$ Mean \pm Standard deviation

BMI : Body Mass Index

\subsection{Measurement}

For the experiment, a typical lecture room chair $(41.5 \mathrm{~cm}$ high, $42 \mathrm{~cm}$ wide, and $37 \mathrm{~cm}$ long) with a backrest but no armrests was used. A goniometer was used to bend the subject's knees $90^{\circ}$ while sitting in the chair. In this position, the subject's two feet were positioned at shoulder width, as measured by a tape measure.

An electromyogram is a bio signal that shows the invisible movement of the muscle during motion and an electromyogram device measures the electronic signal generated in the muscle during motion [18]. The EMG signal is a record of electric signals generated by physiological changes in muscle fiber membranes. These signals are measured by an electromyograph consisting of electrodes, an amplifier, band-pass filter, analog-to-digital conversion unit, and data-recording unit.

To measure the muscle activity of the VL, RF, VM, AT, and GM, a surface electromyogram WEMG-8 (LXM5308, Laxtha Inc., Korea) was used. In total, 11 electrodes of 2,223 $\mathrm{H}$ were used. The sample collection rate of the signal was set at 1,024 $\mathrm{Hz}$ and a $60-\mathrm{Hz}$ notch filter was used. The real-time analysis program TeleScan (LAXTHA Inc., Korea) was used for the signal storage and signal process of the electromyogram (Fig. 1).

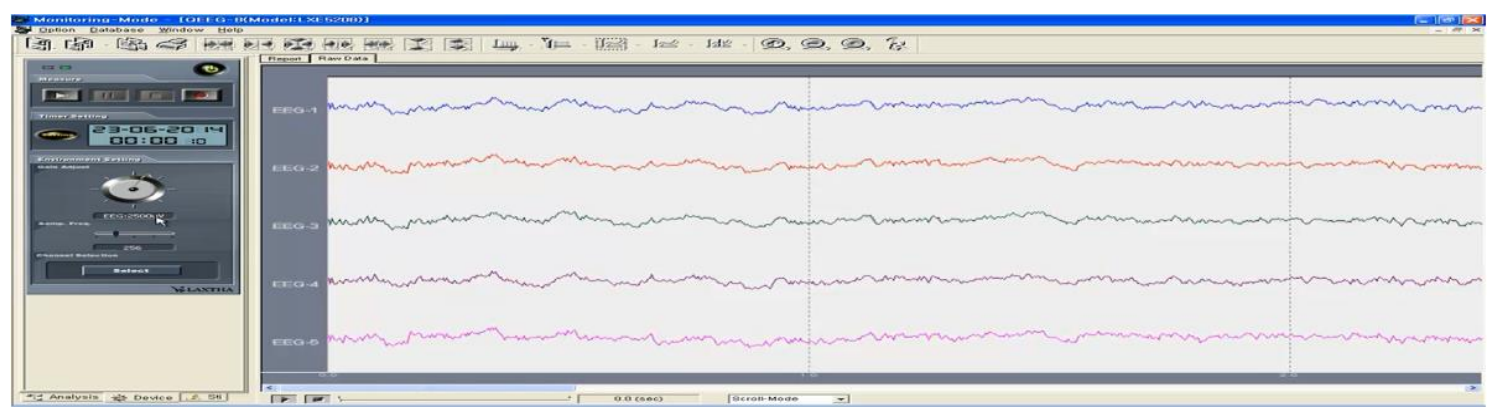

Figure 1. Each Channel in EMG Signal

\section{1) Methods}

For the experiment, a typical lecture room chair $(41.5 \mathrm{~cm}$ high, $42 \mathrm{~cm}$ wide, and $37 \mathrm{~cm}$ long) with a backrest but no armrests was used. A goniometer was used to bend the subject's knees $90^{\circ}$ while sitting in the chair. In this position, the subject's two feet were positioned at shoulder width, as measured by a tape measure. 
For the adjustable-height experiment, an adjustable height chair with no armrests was used. While seated, all subjects bent their hip joint at $90^{\circ}$ and the middle of hips was positioned at the edge of chair. To have consistent measurement posture for all subjects, the angle of the knee joints was set to $90^{\circ}$ by setting the chair height to $80 \%$ of the knee joint height of each subject [7].

Since the femoral length in contact with the chair while seated can affect posture and standing, this length also needed to be consistent. In a previous study a femoral contact portion was set to $50 \%$ of the total femoral length [19]. Through the pre-test, the femoral contact portion was set to $70 \%$ of the femoral length while sitting comfortably. The gap between the feet was set to shoulder width and the angles of the ankle and knee joints were set to $90^{\circ}$ [20]. Then the subject's two arms were placed across the chest in an $\mathrm{X}$ shape to avoid compensation of movement by the.

Using only movements of the trunk and lower extremities, the subject performed the assigned motions while his or her two feet remained at shoulder width. The sitting posture was set as the starting posture and all subjects conducted three STS movements in five seconds. To prevent muscle fatigue, a 10 -sec rest was given between sets. All subjects were informed about electrode attachment and locations. Before the measurement, wires between electrodes and EMG system were arranged neatly and EMG signals on the screen were observed to see any movement noise.

\section{2) EMG Electrode Placement Location}

Prior to attachment of electrodes, body hair in the attachment site was shaved and the skin was cleansed with alcohol gauze to minimize skin resistance. The ground electrode was placed at the $\mathrm{C} 7$ spinous process. To remove noise due to wire movements, Kinesio tape was used to fix the wires in place. Causes of any noise were resolved before placing the electrode. The distance between electrodes was kept to $2 \mathrm{~cm}$, and the electrodes were placed in parallel to the direction of the muscle fibers. The surface electrodes recorded a muscle contraction to mean a change in muscle belly position. (Table 2).

Table 2. Electrode Placement for Detection of Muscle Activation

\begin{tabular}{l} 
Muscle \\
\hline Vastus medialis \\
$\begin{array}{c}\text { The distance along a line from the superior internal side of the patella to the } \\
\text { anterior medial iliac spine, starting from the patella }\end{array}$ \\
The distance along a line from the superior medial side of the patella to the \\
anterior inferior iliac spine, starting from the patella \\
The distance along a line from the superior lateral side of the patella to the \\
anterior superior iliac spine, starting from the patella \\
The percentage distance from the medial side of the popliteus cavity to the \\
medial side of the achilles tendon insertion, starting from the tuberosity of \\
tibia \\
Tibialis anterior \\
The percentage distance from the tuberosity of tibia to the medial malleolus
\end{tabular}

\section{3) EMG Signal Collection}

After the researcher gave the start command, the lower extremity muscles activity was measured as the subject performed three STS movements in five seconds. Data for the first second and last second were removed and the muscle signals over the remaining 
three seconds were averaged. Maximum voluntary isometric contraction (\%MVIC) of muscle activity, a method based on the maximum isometric contraction value of muscle measured at the time of standing, was used in the data analysis [21].

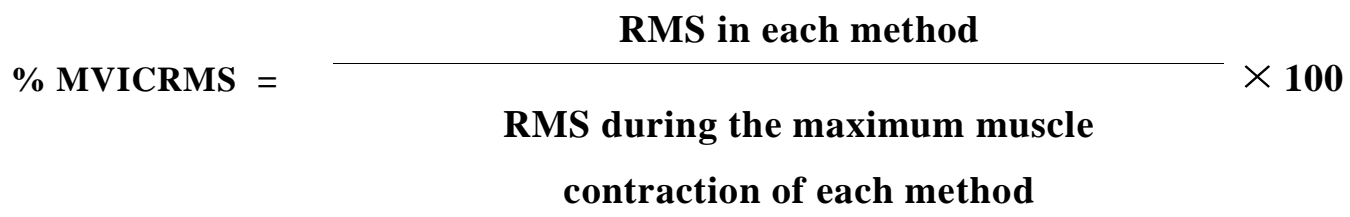

\subsection{Statistical Analysis}

The average and standard deviation were computed for the physical characteristics of each group and the measurement results of each variable. For the data treatment of the study results, the statistics program SPSS ver. 18.0 was used. An independent t-test was performed for the verification of differences according to chair type and gender. A paired t-test was performed for the verification of a difference between the standing and sitting groups. The statistical significance le vel $\alpha$ was set at 0.05 .

\section{Results}

\subsection{Socio-demographic Variables of the Research Subjects}

There was an even number of research samples for each gender, i.e., 26 men and 26 women. As for age, 20-years-olds were the majority, representing $55.8 \%$ of the sample, and as many as $78.8 \%$ of the research subjects regarded their health condition as healthy. As for smoking habit, non-smokers were dominant, representing $75.0 \%$ of the entire sample. The primarily used foot was the right foot, representing $92.3 \%$ of the sample, and the rate of alcohol consumption was mostly once or twice a week, representing $69.2 \%$ of the sample. Regarding family members, the combination of the participant and his/her parents was most common, representing $76.9 \%$ of the sample. As for monthly income, more than $300,000 \mathrm{KRW}$ was the most common rate, representing $48.0 \%$ of the sample (Table 3 ).

Table 3. Socio-demographic Variables of the Subjects $(\mathrm{N}=52)$

\begin{tabular}{llcc}
\hline Category & Division & Frequency & Percentage(\%) \\
\hline \hline \multirow{2}{*}{ Gender } & Male & 26 & 50.0 \\
& Female & 26 & 50.0 \\
\hline \multirow{4}{*}{ Year(s) } & 19 year & 4 & 7.7 \\
& 20 year & 29 & 55.8 \\
& 21 year & 12 & 23.1 \\
& 23 year & 2 & 3.8 \\
& 24 years or more & 5 & 9.6 \\
\hline \multirow{3}{*}{ Health condition } & Good & 41 & 78.8 \\
& Standard & 10 & 19.3 \\
& Weak & 1 & 1.9 \\
\hline Smoking & No & 39 & 75.0 \\
\hline
\end{tabular}




\begin{tabular}{llcc}
\hline & Yes & 13 & 25.0 \\
\hline \multirow{2}{*}{ Main foot } & Left & 4 & 7.7 \\
& Right & 48 & 92.3 \\
\hline \multirow{3}{*}{ Drinking } & No & 13 & 25.0 \\
& Once and twice a week & 36 & 69.2 \\
& More than 3 times a week & 3 & 5.8 \\
\hline \multirow{2}{*}{ Family } & Myself & 2 & 3.8 \\
& Myself with parents & 40 & 76.9 \\
& Myself with brothers & 10 & 19.3 \\
\hline \multirow{3}{*}{ Monthly income } & Less than 100,000 won & 12 & 23.1 \\
& Less than 200,000 won & 3 & 5.8 \\
& Less than 300,000 won & 12 & 23.1 \\
\hline \hline Total & More than 300,000 won & 25 & 48.0 \\
\hline
\end{tabular}

\subsection{Comparison of Muscle Activity According to Chair Type}

The muscle strength of the lower limbs increases more in the case of a small STS movements value according to chair type. There was no significant difference in a comparison of different chair types ( $p>.05$ ). As for the VM, RF, and VL, the muscle activity increased more in the case of a fixed chair than a wheeled chair in both groups. The muscle activity of the GC and AT increased more in the case of a wheeled chair in both the standing and sitting groups and in the standing group, respectively, while the muscle activity of the AT increased more in the case of a fixed chair in the sitting group (Table 4) (Fig. 2).

Table 4. A Comparison of Muscle Activity According to Posture between Fixed Chair and Wheeled Chair in each Group (Unit : \%MVIC)

\begin{tabular}{clllll}
\hline \multirow{2}{*}{ Muscle } & Posture & $\begin{array}{l}\text { Fixed Chair } \\
(\mathrm{n}=52)\end{array}$ & $\begin{array}{l}\text { Wheeled } \\
\text { Chair } \\
(\mathrm{n}=52)\end{array}$ & $t$ & $p$ \\
\hline \hline \multirow{2}{*}{ VM } & Standing & $23.94 \pm 9.56$ & $23.14 \pm 9.80$ & .42 & .674 \\
\cline { 2 - 6 } & Sitting & $19.96 \pm 9.81$ & $19.20 \pm 9.07$ & .41 & .682 \\
\hline \multirow{2}{*}{ RF } & Standing & $23.95 \pm 13.59$ & $23.05 \pm 12.69$ & .35 & .729 \\
\cline { 2 - 6 } & Sitting & $22.85 \pm 12.91$ & $21.28 \pm 11.63$ & .65 & .516 \\
\hline \multirow{2}{*}{ VL } & Standing & $28.07 \pm 12.57$ & $27.56 \pm 14.07$ & .20 & .845 \\
\cline { 2 - 6 } & Sitting & $23.46 \pm 12.76$ & $22.48 \pm 12.53$ & .39 & .694 \\
\hline \multirow{2}{*}{ GC } & Standing & $15.99 \pm 14.03$ & $16.75 \pm 15.59$ & -.26 & .495 \\
\cline { 2 - 6 } & Sitting & $16.50 \pm 14.79$ & $19.69 \pm 25.39$ & -.78 & .821 \\
\hline \multirow{2}{*}{ TA } & Standing & $21.91 \pm 21.38$ & $22.93 \pm 24.16$ & -.23 & .710 \\
\cline { 2 - 6 } & Sitting & $27.59 \pm 38.88$ & $25.22 \pm 24.48$ & .37 & \\
\hline
\end{tabular}

Mean \pm SD: Mean \pm Standard deviation

VM : Vastus medialis, RF : Rectus femoris, VL : Vastus lateralis

GC : Gastrocnemius, TA : Tibialis anterior 


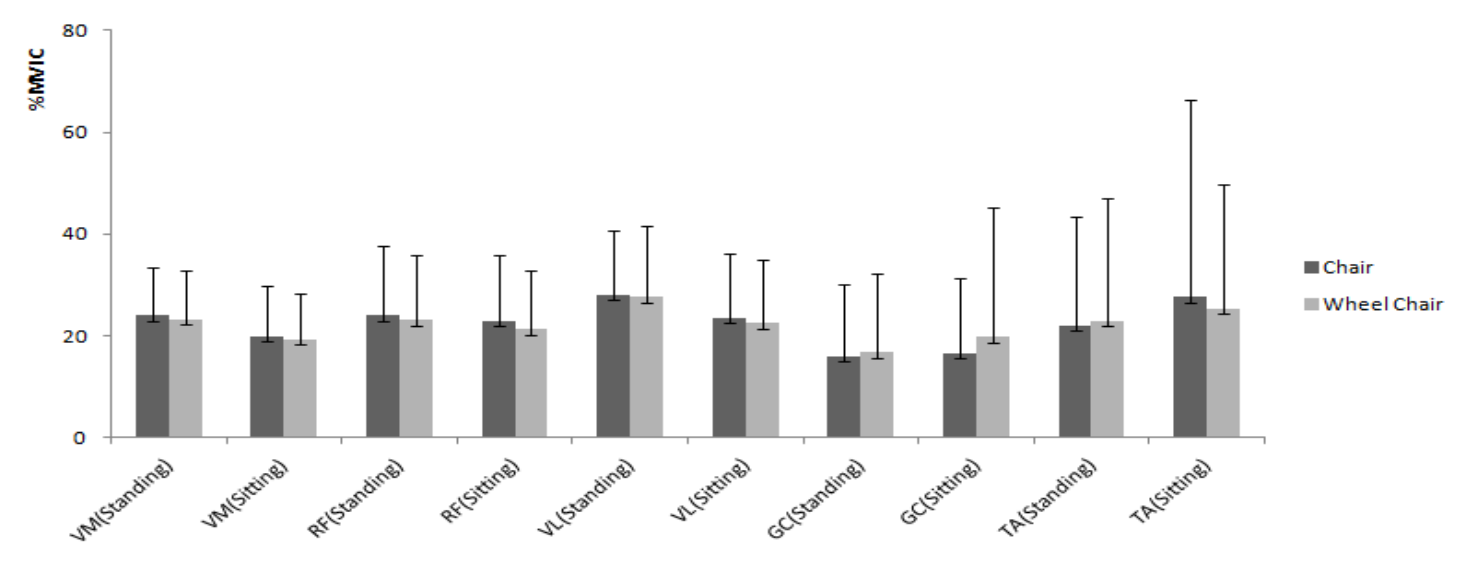

Figure 2. Comparison of Fixed Chair or Wheeled Chair Distribution

\subsection{Comparison of Muscle Activity According to Posture Type}

The muscle activity of the VM increased more in the case of a standing position than a sitting position. The RF and VL also showed greater increases in muscle activity in a standing position than a sitting position. On the contrary, the GC and AT showed greater decreases in muscle activity in a standing position than a sitting position (Table 5) (Fig. 3).

Table 5. A Comparison of Muscle Activity According to Posture between Standing and Sitting in Each Group (Unit : \%MVIC)

\begin{tabular}{|c|c|c|c|c|}
\hline \multirow{2}{*}{ Muscle } & \multicolumn{2}{|c|}{ Group } & \multirow{2}{*}{$t$} & \multirow{2}{*}{$p$} \\
\hline & Standing $(n=52)$ & Sitting $(n=52)$ & & \\
\hline VM & $23.54 \pm 9.64$ & $19.58 \pm 9.41$ & 7.76 & $.000 *$ \\
\hline $\mathrm{RF}$ & $23.50 \pm 13.10$ & $22.07 \pm 12.25$ & 2.06 & $.042 *$ \\
\hline VL & $27.82 \pm 13.28$ & $22.97 \pm 12.59$ & 8.36 & $.000^{*}$ \\
\hline $\mathrm{GC}$ & $16.37 \pm 14.76$ & $18.10 \pm 20.74$ & -1.55 & .124 \\
\hline TA & $22.42 \pm 22.68$ & $26.41 \pm 32.35$ & -2.59 & $.011 *$ \\
\hline
\end{tabular}

$* \mathrm{p}<.05$

Mean \pm SD : Mean \pm Standard deviation

VM : Vastus medialis, RF : Rectus femoris, VL : Vastus lateralis

GC : Gastrocnemius, TA : Tibialis anterior

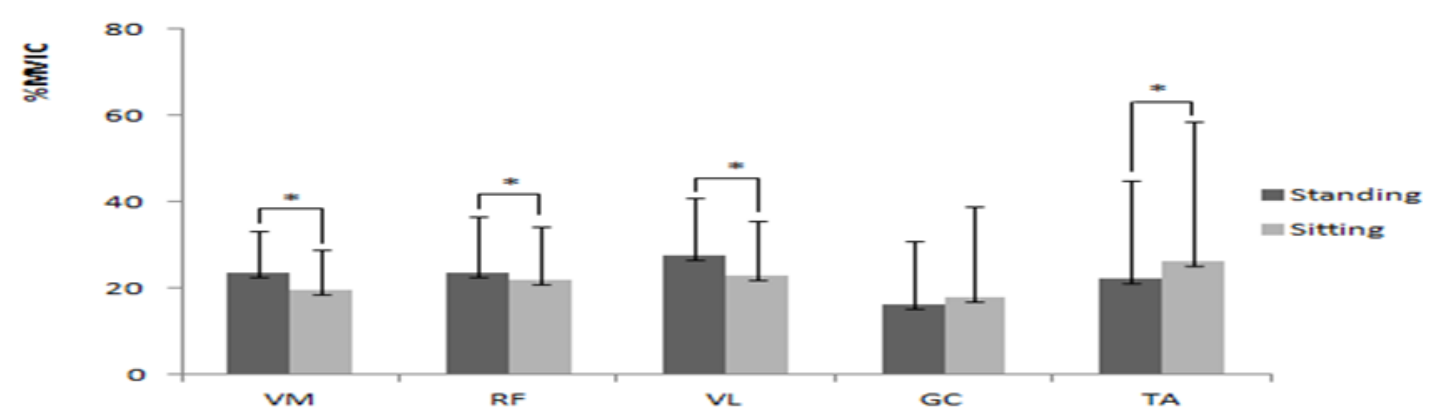

Figure 3. Comparison of STS Posture Distribution 


\section{Discussion}

The STS movement is a functional task that is most commonly required in daily life. Moreover, the STS movement is an important motion that precedes functional activities, such as walking [22]. It is also a practical evaluation method frequently used in technical research and rehabilitation studies [23].

An STS movement test consists of measurements taken while performing sitting and standing motions to assess lower extremity muscle strength and balance. In one study, STS test was employed to determine death rate of the elderly in association with disability prediction and changes in physical performance due to degradation in balance [24]. STS-movement research is mainly classified into three subjects: characteristics of subjects, strategies, and chair type [25]. However, few studies have been done on chair height under the same test conditions.

This study used EMG (WEMG-8) to determine muscle activity in the lower extremities during STS movements from fixed-leg and wheel-leg chairs of the same shape with 52 subjects (26 males, 26 females). While standing from a fixed-leg chair, according to chair type and gender, a significant difference was seen in rectus femoris and vastus lateralis and while sitting, a significant difference was seen in vastus medialis, rectus femoris, and vastus lateralis $(\mathrm{p}<0.05)$. Regardless of differences in height and leg length of the subjects, no significant difference was seen between groups because daily activities and diet were not controlled.

Brunt [26] reported that activities in the tibialis anterior and rectus femoris were higher when standing with one foot positioned further back than the other foot than when standing with both feet symmetrically positioned.

This study limited the ankle joint at 90 degrees; hence, it differs from previous literature that investigated the STS movement's impact on the muscle activity of the lower limb muscles according to the changing location of the feet. When stand-to-sit and STS motions were performed with the knee joints at 90 degrees, the GM and RF did not show significant differences ( $p>.05$ ), while the VM, VL, and AT showed significant differences $(\mathrm{p}<.05)$.

Cheng et al. [27] argued the AT is activated most often before lifting the hip during the STS movement, and it is a crucial factor for maintaining ankle stability. Lomaglio et al. [15] reported the RF, VL, and VM are among the muscles that are activated most during the STS movement.

As for the stand-to-sit movement, depending on the chair type, the VM, RF, VL, GM, and AT showed no significant differences in lower limb muscle activity during the stand-to-sit movement between a fixed chair and a wheeled chair ( $p>.05)$.

Cahill et al. [22] reported that the dynamic stability of the quadriceps muscle, GM, and soleus muscle is important for the STS movement, as they play a critical role in controlling motion speed. Moreover, Eriksrud et al. [28] reported the RF achieves knee stabilization prior to hip joint flexion and knee extension during the STS movement.

Carr and Shepherd [29] reported that the dynamic stability of the quadriceps femoris, gastrocnemius, and soleus muscles was important to the standing motion, and these muscles played an important role in controlling motion speed. Furthermore, Eriksrud and Bohannon [28] reported that stability in knees was done before hip joint flexure at rectus femoris and knee extension while performing standing motion.

This study had several limitations. The number of subjects was too small, and the study results cannot be generalized. Second, the safety of the subjects was the foremost concern in this study, although the subjects had different ages, physical 
ability, and functions. Third, the chair's size did not match the physical characteristics (sitting height, physical height) of all the test subjects.

In this study, there was no significant difference between a fixed chair and a wheeled chair when the STS movement was performed on a stable, supportive surface. However, if the controlled condition that makes a supportive surface unstable, as well as diverse dependent variables are included in future studies, it can be useful for providing fundamental data regarding the muscle activation of lower limbs according to chair type. In future studies, it will be necessary to build a strong power of verification that can conform to the purpose of the study by using a greater number of research subjects and various evaluation tools.

\section{Conclusion}

STS movement can be used as a health marker in patients and the elderly. A significant difference was seen in some muscle groups while performing standing and sitting motions from a fixed-leg chair. In a future study, subjects with a wider range of ages and occupations, such as office and field workers, will be included so that appropriate strength training programs can be developed that factor in the unique needs of various job fields. In addition, a comparative study of the mental state between the healthy subjects and those with physical or medical limitations will be added.

\section{References}

[1] Y. J. Yu, "Kinetic analysis of sit-to-stand movement in stroke patients", The Korean Journal of Physical Education, vol. 43, (2004), pp. 521-529.

[2] R. Hardy, R. Cooper, I. Shah, S. Harridge, J. Guralnik and D. Kuh, "Is chair rise performance a useful measure of leg power", Aging Clin Exp Res., vol. 22, (2010), pp. 412-418.

[3] M. Galli, V. Cimolin, M. Crivellini and I. Campanini, "Quantitative analysis of sit to stand movement,experimental set-up definition and application to healthy and hemiplegic adults", Gait Posture, vol. 28, (2008), pp. 80-85.

[4] M. E. Roebroeck, C. A. Doorenbosch, J. Harlaar, R. Jacobs and G. J. Lankhorst, "Biomechanics and muscular activity during sit-to-stand transfer", Clin Biomech (Bristol, Avon), vol. 9, (1994), pp. 235-244.

[5] A. Onell, "The vertical ground reaction force for analysis of balance", Gait Posture, vol. 12, (2000), pp. 13-17.

[6] M. J. Pavol and Y. C. Pai, "Deficient limb support is a major contributor to age differences in falling", Journal of Biomechanics, vol. 40, (2007), pp. 1325-1318.

[7] M. Schenkman, R. A. Berger, P. O. Riley, R. W. Mann and W. A. Hodge, "Whole-body movements during rising to standing from sitting", Phys Ther., vol. 70, (1990), pp. 638-648.

[8] M. A. Hughes, D. K. Weiner, M. L. Schenkman, R. M. Long and S. A. Studenski, "Chair rise strategies in the elderly", Clin Biomech., vol. 9, (1994), pp. 187-192.

[9] P. O. Riley, M. L. Schenkman, R. W. Mann and W. A. Hodge, "Mechanics of a constrained chair-rise", J Biomech., vol. 24, (1991), pp. 77-85.

[10] K. M. Kerr, J. A. White and D. A. Barr, "Analysis of the sit-stand-sit movement cycle in normal subjects", Clin Biomech., vol. 12, (1997), pp. 236-245.

[11] S. Edwards, "Neurological physiotherapy, A problem-solving approach", Churchill Livingstone, (2002).

[12] B. Yu, N. Holly-Crichlow, P. Brichta, G. R. Reeves, C. M.Zablotny and D. A. Nawoczenski, "The effects of the lower extremity joint motions on the total body motion in sit-to-stand movement", Clin Biomech (Bristol, Avon), vol. 15, (2000), pp. 449-455.

[13] N. Maeda, J. Kato, K. Itotani, K. Onishi and M. Murakami, "Relationship between sit-to-stand (STS) motion characteristics and walking ability in stroke patients", Gait \& Posture, vol. 36, (2012), pp. S57-S58.

[14] S. W. Chou, A. M. Wong, C. P. Leong, W. S. Hong, F. T. Tang and T. H. Lin, "Postural control during sit to stand and gait in stroke patient”, Am J Phys Med Rehabil., vol. 82, (2003), pp. 4247.

[15] M. J. Lomaglio and J. J. Eng, "Muscle strength and weight-bearing symmetry relate to sit-tostand performance in individuals with stroke", Gait Posture, vol. 22, (2005), pp. 126-131.

[16] J. P. Scholz, D. Reisman and G. Schöner, "Effects of varying task constraints on solutions to joint coordination in a sit-to-stand task", Exp Brain Res., vol. 141, (2001), pp. 485-500.

[17] J. R. Cram, G. S. Kasman and J. Holtz, "Introduction to Surface Electromyography", (1998). 
[18] Y. J. Choi and H. J. Yu, "Human arm motion tracking base dons EMG signal", Journal of Control, Automation, and Systems Engineering, vol. 13, (2007), pp. 769-776.

[19] Y. B. Seven, N. E. Akalan and C. A. Yucesoy, "Effects of back loading on the biomechanics of sit-to-stand motion in healthy children", Hum Mov Sci., vol. 27, (2008), pp. 79-65.

[20] T. Yamada and S. Demura, "Influence of the relative difference in chair seat height according to different lower thigh length on floor reaction force and lower-limb strength during sit-to-stand movement”, J Physiol Anthropol Appl Human Sci., vol. 23, (2004), pp. 203-197.

[21] E. Criswell, "Cram's introduction to surface electromyography”, Jones \& Bartlett Learning, (2010).

[22] B. M. Cahill and J. H. Carr, "Inter-segmental co-ordination in sit-to-stand; an age cross-sectional study", Physiother Reserch International, vol. 4, (1999), pp. 12-27.

[23] K. K. Schind, E. Uher and F. Kainberger, "Long-term home exercise program, Effect in women at high risk of fracture", Archives of physical medicine and rehabilitation, vol. 81, (2000), pp. 319-319.

[24] S. L. Whitney, D. M. Wrisley, G. F. Marchetti, M. A. Gee, M. S. Redfern and J. M. Furman, "Clinical measurement of sit-to-stand performance in people with balance disorders", Validity of Data for the Five-Times-Sit-to-Stand Test, Physical Therapy, vol. 85, (2005), pp. 1045-1034.

[25] W. G. Janssen, H. B. Bussmann and H. J. Stam, "Determinants of the sit-to-stand movement", A review, Phys Ther., vol. 82, (2002), pp. 866-879.

[26] D. Brunt, B. Greenberg, S. Wankadia, M. A. Trimble and O. Shechtman, "The effect of foot placement on sit to stand in healthy young subjects and patients with hemiplegia", Arch Phys Med Rehabil., vol. 83, (2002), pp. 924-929.

[27] P. T. Cheng, C. L. Chen, C. M. Wang and W. H. Hong, "Leg muscle activation patterns of sit-tostand movement in stroke patients", Am J Phys Med Rehabil., vol. 83, (2004), pp. 10-16.

[28] O. Eriksrud and R. W. Bohannon, "Relationship of knee extension force to independence in sit to stand performance in patients receiving acute rehabilitation", Phys Ther., vol. 83, (2003), pp. $551-551$

[29] H. Janet, M. A. Carr, B. Roberta and M. A. Shepherd, "Stroke Rehabilitation; guidelines for exercise and training to optimize motor skill”, Manual Therapy, vol. 8, (2003), pp. 261-268.

\section{Authors}

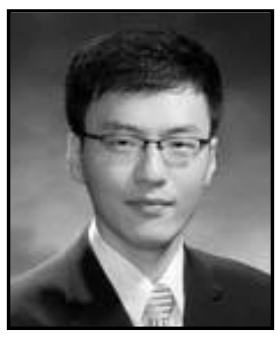

Sunghyoun Cho, he received the $\mathrm{PhD}$ degree in Physical Therapy from Daegu University. He is a professor in Department of Physical Therapy in Nambu University, Gwangju, Republic of Korea. His recent interests focus on Biomechanics and Therapeutic exercise.

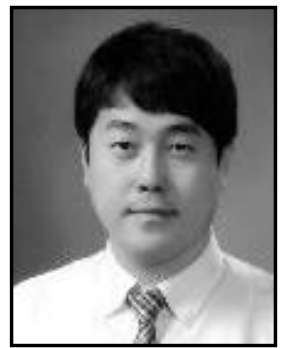

Haewon Byeon, he received the DrSc degree in Biomedical Science from Ajou University School of Medicine. He is a professor in Department of Speech Language Pathology \& Audiology and director of Speech Language Pathology Center in Nambu University, Gwangju, Republic of Korea. His recent interests focus on mild cognitive impairment and motor speech disorders. 